\title{
Amniotic Epithelial Cells Accelerate Diabetic Wound Healing by Modulating Inflammation and Promoting Neovascularization
}

\author{
Yongjun Zheng, ${ }^{1}$ Shiqing Zheng, ${ }^{1,2}$ Xiaoming Fan, ${ }^{1}$ Li Li, ${ }^{3}$ Yongqiang Xiao, ${ }^{1}$ Pengfei Luo, ${ }^{1}$ \\ Yingying Liu, ${ }^{1}$ Li Wang, ${ }^{1}$ Zhenci Cui, ${ }^{1}$ Fei He, ${ }^{1}$ Yuhuan Liu $\mathbb{D},{ }^{3}$ Shichu Xiao $\mathbb{D}^{1},{ }^{1}$ \\ and Zhaofan Xia (iD ${ }^{1}$ \\ ${ }^{1}$ Burns Center, Changhai Hospital, Second Military Medical University, Shanghai, China \\ ${ }^{2}$ No. 73051 Troop of the Chinese People's Liberation Army, Xiamen, China \\ ${ }^{3}$ Department of Obstetrics and Gynecology, Changhai Hospital, Second Military Medical University, Shanghai, China
}

Correspondence should be addressed to Yuhuan Liu; 13651968369@163.com, Shichu Xiao; huangzhuoxiao@sohu.com, and Zhaofan Xia; xiazhaofan_smmu@163.com

Yongjun Zheng, Shiqing Zheng, Xiaoming Fan, and Li Li contributed equally to this work.

Received 3 March 2018; Revised 17 May 2018; Accepted 10 June 2018; Published 19 August 2018

Academic Editor: Isotta Chimenti

Copyright ( 2018 Yongjun Zheng et al. This is an open access article distributed under the Creative Commons Attribution License, which permits unrestricted use, distribution, and reproduction in any medium, provided the original work is properly cited.

\begin{abstract}
Human amniotic epithelial cells (hAECs) are nontumorigenic, highly abundant, and low immunogenic and possess multipotent differentiation ability, which make them become ideal alternative stem cell source for regenerative medicine. Previous studies have demonstrated the therapeutic potential of hAECs in many tissue repairs. However, the therapeutic effect of hAECs on diabetic wound healing is still unknown. In this study, we injected hAECs intradermally around the full-thickness excisional skin wounds of $\mathrm{db} / \mathrm{db}$ mice and found that hAECs significantly accelerated diabetic wound healing and granulation tissue formation. To explore the underlying mechanisms, we measured inflammation and neovascularization in diabetic wounds. hAECs could modulate macrophage phenotype toward M2 macrophage, promote switch from proinflammatory status to prohealing status of wounds, and increase capillary density in diabetic wounds. Furthermore, we found that the hAECconditioned medium promoted macrophage polarization toward M2 phenotype and facilitated migration, proliferation, and tube formation of endothelial cells through in vitro experiments. Taken together, we first reported that hAECs could promote diabetic wound healing, at least partially, through paracrine effects to regulate inflammation and promote neovascularization.
\end{abstract}

\section{Introduction}

Diabetic ulcers are a severe, resistant complication of diabetes mellitus (DM), and about one fourth of DM patients endure diabetic lower extremity ulcers during their whole lives [1]. Diabetic wounds tend to heal slowly and be frequently recurrent, not only leading to increasing cost of medical care but also seriously impairing the quality of life in diabetic patients. Current standard treatments in clinics such as debridement, moist dressings, infection control, or wound offloading have not achieved the satisfactory results. Therefore, we urgently need more effective therapeutic approaches to promote diabetic wound healing.
Stem cells have shown great potential in repairing damaged tissue. Mesenchymal stem cell (MSC) is one of the most widely studied stem cells for wound healing, and several studies have demonstrated inspiring preclinical results through animal models [2-5]. Nonetheless, several arguments still exist against the widely use of MSC in clinics, including the probability of tumorigenesis and low cell production from MSC harvesting. Human amniotic epithelial cells (hAECs) are derived from the amniotic membrane and demonstrate a potential source of stem cells. These cells are nontumorigenic and low immunogenic and possess multipotent differentiation ability [6-8]. Furthermore, hAECs are highly plentiful, and about $1.5 \times 10^{8}$ cells could be separated 
from each amniotic membrane, which is plenty for using hAECs directly in clinics without amplification in vitro [9]. In this regard, the use of hAECs for regenerative medicine holds great promise.

Recently, the beneficial effects of hAECs in many tissue repairs are reported [10-13]. Although studies have shown that hAECs play their beneficial roles through preventing inflammatory responses, the exact mechanisms of hAECs for promoting tissue repairs remain unclear $[11,14]$. A common feature of refractory healing wound is persistent inflammation, impaired vascularization, and decreased expression of growth factors [15]. Whether hAECs play a role in diabetic wound healing associated with inflammation and neovascularization is still unknown.

In this study, we determined whether hAEC transplantation could promote diabetic wound healing by injecting hAECs intradermally around the full-thickness excisional skin wounds of $\mathrm{db} / \mathrm{db}$ mice. We further explored related mechanisms through in vivo and in vitro experiments, mainly focusing on inflammation and neovascularization.

\section{Materials and Methods}

2.1. Cell Isolation and Culture. All research proposals were permitted by the Ethics Committee of Changhai Hospital, Shanghai, China. We first obtained informed consent from parturients and then got placentas directly from parturients immediately after cesarean section. All donors had no human hepatitis B and C, syphilis, and HIV verified by serological tests. Collection and isolation of hAECs was performed as described previously [9]. hAECs were cultured in a highglucose DMEM supplemented with 10\% FBS (complete medium), and hAECs at passage 2-3 were used.

Mouse macrophages were separated from bone marrow of wild-type C57BL/6 mice and cultured in a complete medium, and primary-cultured macrophages were used for further cell experiments as previously described [16]. Human umbilical vein endothelial cells (HUVECs) were purchased from ScienCell (San Diego, USA) and cultured in the endothelial cell medium (ECM, Gibco, Life Technologies) supplemented with $10 \%$ FBS, and HUVECs between passages 2 and 4 were used for further in vitro experiments. To assess the effects of the hAEC-conditioned medium (hAEC-CM) on macrophage phenotype switch in vitro, we used IFN- $\gamma$ and TNF- $\alpha$ (20 ng/ml each, R\&D Systems) to stimulate the macrophages for 24 hours with or without hAEC-CM. Complete medium without TNF- $\alpha$ and IFN- $\gamma$ was used as control.

2.2. Preparation of the hAEC-Conditioned Medium (hAEC$C M$ ). We prepared hAEC-CM using a method analogous to the one described previously [17]. In brief, hAECs were digested with trypsin and then $2 \times 10^{6}$ hAECs were cultured in a $100 \mathrm{~mm}$ plate overnight. We changed the complete culture medium into a $10 \mathrm{ml}$ serum-free high-glucose DMEM and cultured for another 24 hours. The supernatant was collected and concentrated to 10 times the concentration of the collected hAEC-CM as the final hAEC-CM by using Amicon Ultra-15 ultrafiltration conical tubes (Millipore, $3 \mathrm{kDa}$ ).
2.3. Animal Model and Treatment. All animal protocols were complied with the rules of the animal use and care committee of Changhai Hospital, Shanghai, China. The $\mathrm{db} / \mathrm{db}$ mouse was a widely accepted animal model of type 2 diabetes. We purchased the male and $8-12$ weeks old $\mathrm{db} / \mathrm{db}$ mouse (C57BL/KsJ, leptin receptor-deficient diabetes) from SLAC Laboratory Animal Co. Ltd., Shanghai, China. Only mice with a $>300 \mathrm{mg} / \mathrm{dl}$ blood glucose level were included for further animal experiments. Mice were anesthetized, and then two symmetric splint wounds $(10 \mathrm{~mm}$ diameter $)$ with fullthickness skin defect were made on the back of each mouse based on the previous study [18]. We divided the mice equally into 2 groups: hAEC group and blank group (PBS group). $1 \times 10^{6}$ cells suspended in $100 \mu$ l PBS were injected intradermally around the wound. Photographs of wounds were taken regularly, and then, the wound healing rate was figured by Image-Pro Plus software.

2.4. Skin Histological Analysis and Immunohistochemistry. The wounds with the margins were excised at day 10 after wounding, fixed in 10\% formalin, and then embedded in paraffin for hematoxylin and eosin (H\&E) staining and immunohistochemistry staining. We measured the wound bed area of each section stained with $\mathrm{H} \& \mathrm{E}$ using Image-Pro Plus software. Angiogenesis in day 10 wounds was observed by immunohistochemistry using CD31 (Santa Cruz, USA) as the primary antibody and then stained with DAB (Thermo, USA). For evaluation the number of M1 and M2 macrophages in day 10 wounds, we counterstained the sections by immunofluorescence with CD68 (Abcam) and iNOS (Abcam) to calculate M1 macrophages and with CD68 and CD206 (Abcam) to calculate M2 macrophages. All sections were finally stained with DAPI.

2.5. ELISA. Wounds with the margins at day 10 were excised with a skin biopsy punch and homogenized in cold PBS by a Dounce homogenizer. After being sonicated, the homogenates were centrifuged at $4^{\circ} \mathrm{C}(10,000 \mathrm{rpm}, 20$ minutes). Supernatants were collected, and expression levels of IL-6, IL- $1 \beta$, TNF- $\alpha$, VEGF, TGF- $\beta 1$, and IGF- 1 were calculated by ELISA. All ELISA kits were purchased from R\&D Systems and used according to the manufacturer's instruction.

2.6. HUVEC Proliferation Assay. Proliferation of HUVECs was evaluated by a CCK-8 Kit (Liankebio, China) in conformity to the instruction of the manufacturer. In brief, HUVECs were digested with trypsin, and $5 \times 10^{3}$ cells were plated into each well of a 96-well plate (BD Falcon). After being cultured in a complete medium for 24 hours, the cells were then cultured in hAEC-CM for another 72 hours. Complete medium was treated as a positive control medium, and serum-free high-glucose DMEM was used as a negative control medium.

2.7. HUVEC Tube Formation Assay. We performed the tube formation assay in conformity to the instructions of the manufacturer. Matrigel (BD Biosciences, USA) was diluted with serum-free DMEM to $5 \mathrm{mg} / \mathrm{ml}$, and $50 \mu \mathrm{l}$ gel was plated into each well of a 96-well plate. $4 \times 10^{4}$ HUVECs suspended in $100 \mu \mathrm{l}$ hAEC-CM were plated into each well. After 


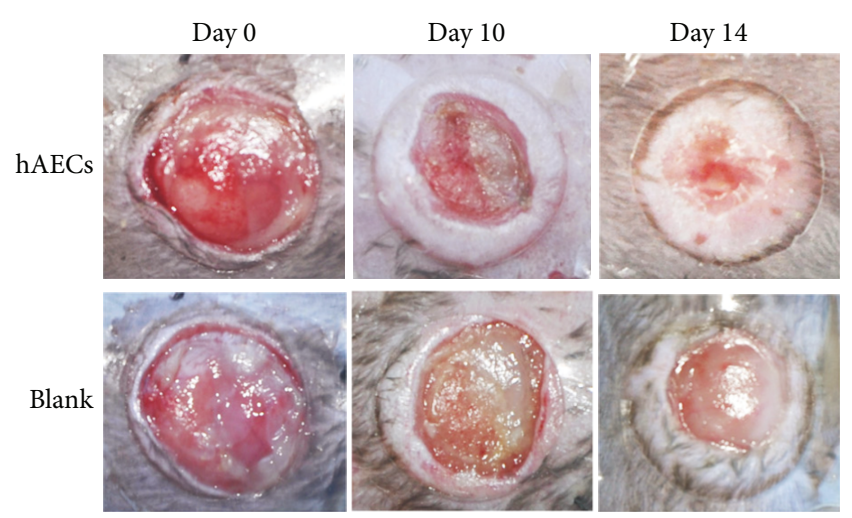

(a)

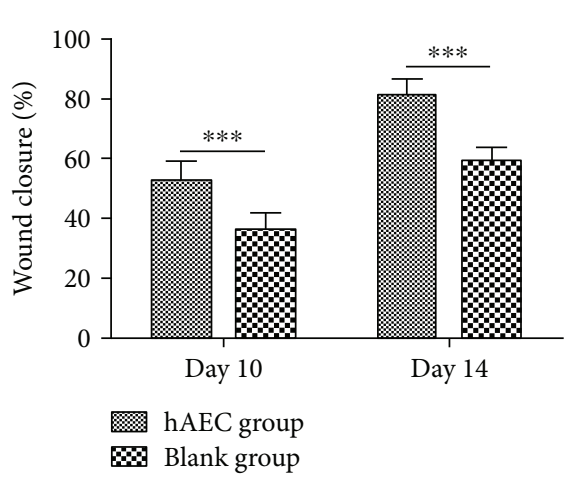

(b)

FIgURE 1: Topical administration of hAECs accelerated diabetic wound healing. (a) Diabetic wounds were treated with hAECs or PBS, examined at day 0, day 10, and day 14 after wounding, and then digitally photographed. (b) Quantitative analysis of wound closure demonstrating a higher wound healing rate in the hAEC-treated group. Data was shown as means $\pm \mathrm{SD} ; n=6 ;{ }^{* * *} P<0.001$.

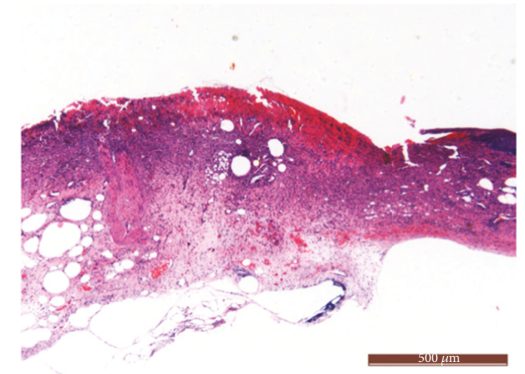

(a)

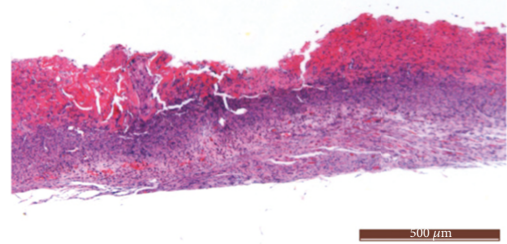

(b)

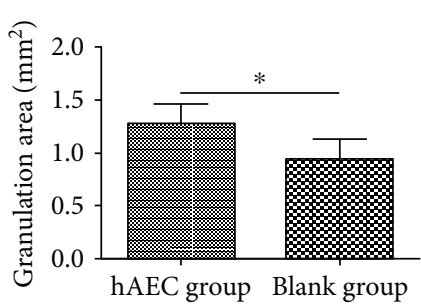

(c)

FIgURE 2: Histomorphometric analysis of diabetic wounds. Representative H\&E staining of wound sections at day 10 from the hAEC group (a) and PBS group (b). Quantitative analysis of the granulation tissue area, showing the increased granulation tissue formation in the hAECtreated group (c). Data was shown as means $\pm \mathrm{SD} ; n=6$; ${ }^{*} P<0.05$; scale bar $=500 \mu \mathrm{m}$.

incubating HUVECs for 6 hours, we took photographs with a microscope system (Leica, Germany) and then calculated the capillary-like structures. Complete medium was treated as a positive control medium, and serum-free high-glucose DMEM was used as a negative control medium.

2.8. HUVEC Migration Assay. We performed HUVEC migration assay by a transwell chamber with $8 \mu \mathrm{m}$ pore size (BD Biosciences, USA). The upper chamber was plated with $1 \times 10^{5}$ HUVECs suspended in serum-free DMEM, and the bottom chamber was added with $600 \mu \mathrm{l}$ hAEC-CM. After 24 hours, we fixed the filters with $10 \%$ formalin, stained them with $0.1 \%$ crystal violet (Sigma, USA), and then counted the migrated cells by Leica QWin image analysis software. Complete medium was treated as a positive control medium, and serum-free high-glucose DMEM was used as a negative control medium.

2.9. Western Blot Analysis. The macrophages were lysed in lysis buffer after washing with ice cold PBS buffer. Lysates were centrifuged, and the supernatants were collected for Western blot analysis. The protein concentration was determined by the BCA protein assay kit. Western blot analysis was performed with primary antibody against iNOS, CD206, and GAPDH as previously described [19].
2.10. Detection of Growth Factors and Inflammatory Cytokines in hAEC-CM. hAEC-CM was collected to detect the expression of growth factors and inflammatory cytokines by using human growth factor antibody array G1 and human inflammation antibody array G1 (RayBiotech Inc.), respectively, in accordance with the manufacturer's protocol.

2.11. Statistical Analysis. We expressed the results as mean \pm $\mathrm{SD}$ and analyzed the data using two-tailed Student's $t$-test or one-way ANOVA by SPSS 16.0. $P<0.05$ was considered as statistical significance.

\section{Results}

3.1. Topical Administration of hAECs Accelerated Diabetic Wound Healing. We treated diabetic wounds with hAECs or PBS and then examined at day 0, day 10, and day 14 after wounding (Figure 1(a)). At day 10, the wound healing rate was significantly higher in the hAEC-treated group (52.16 $\pm 6.80 \%)$ than that in the PBS-treated group $(35.76 \pm 6.19 \%$, $P<0.001$, Figure 1(b)). The gap of the wound healing rate between the two groups gradually increased along with time. Furthermore, we performed H\&E staining (Figures 2(a) and 2(b)) and found that the granulation tissue area was 


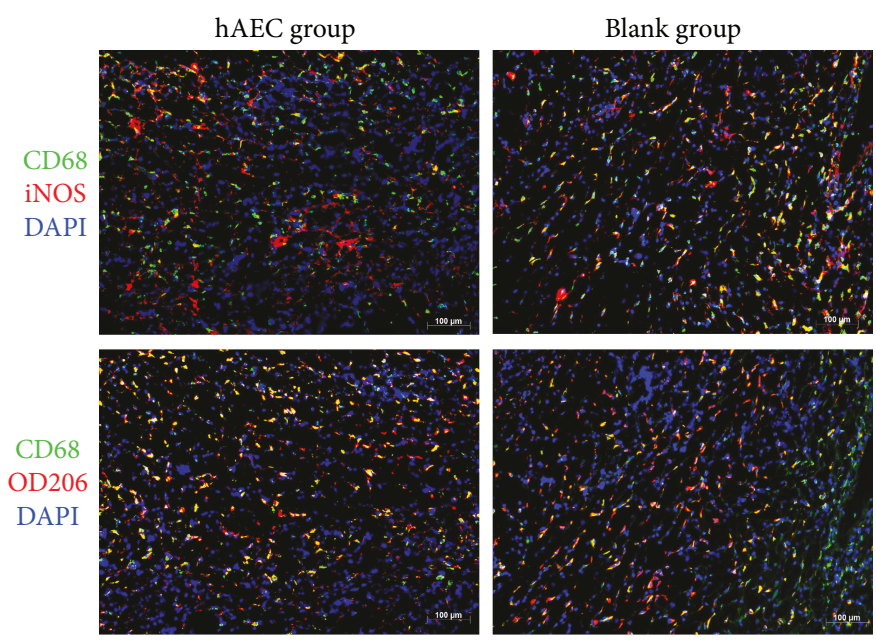

(a)

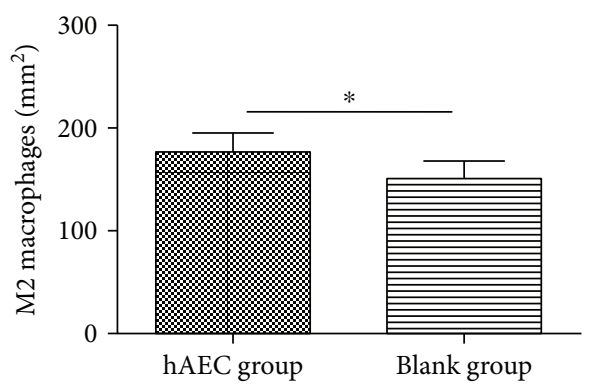

(c)

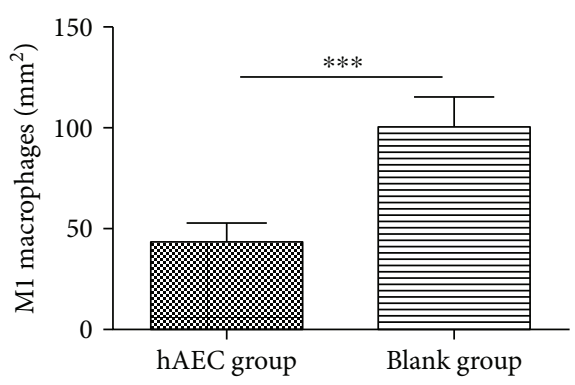

(b)

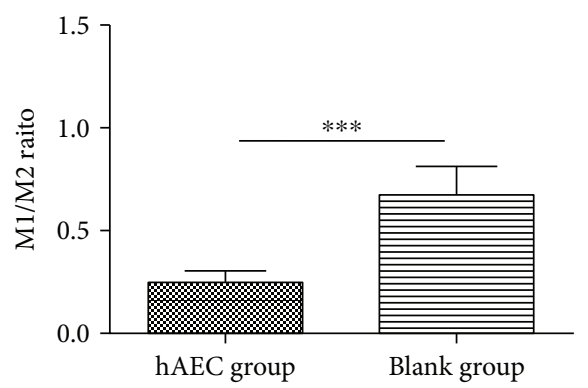

(d)

FIGURE 3: Macrophage phenotype was polarized toward M2 macrophage in the hAEC-treated group. (a) Representative immunofluorescent staining of M1 and M2 macrophages in day 10 wounds. M1 macrophages were shown in the upper panel to be yellow as CD68 ${ }^{+} \mathrm{iNOS}^{+} \mathrm{DAPI}{ }^{+}$ triple-positive staining, and M2 macrophages were shown in the lower panel to be yellow as $\mathrm{CD} 68^{+} \mathrm{CD} 206^{+} \mathrm{DAPI}{ }^{+}$triple-positive staining. (b, c, and d) The number of M1 macrophages was significantly reduced in the hAEC group (b), whereas the number of M2 macrophages was increased (c), resulting in a lower M1/M2 ratio (d). Data was shown as means $\pm \mathrm{SD} ; n=6 ;{ }^{*} P<0.05 ;{ }^{* * *} P<0.001 ;$ scale bar $=100 \mu \mathrm{m}$.
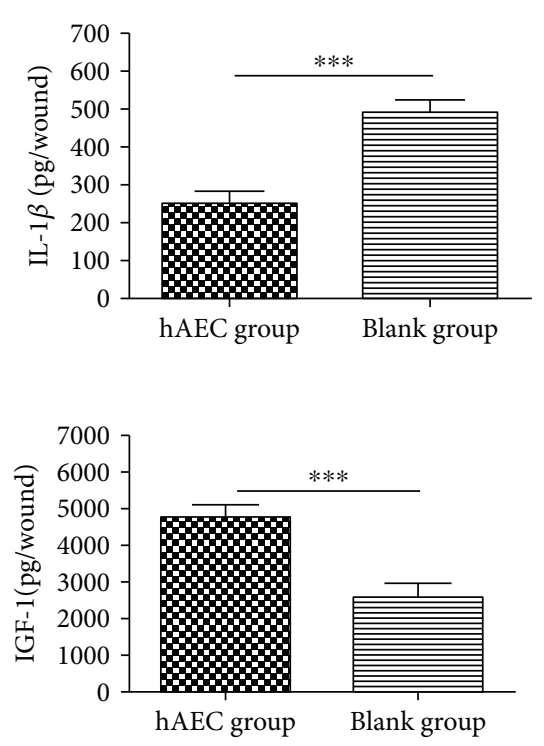
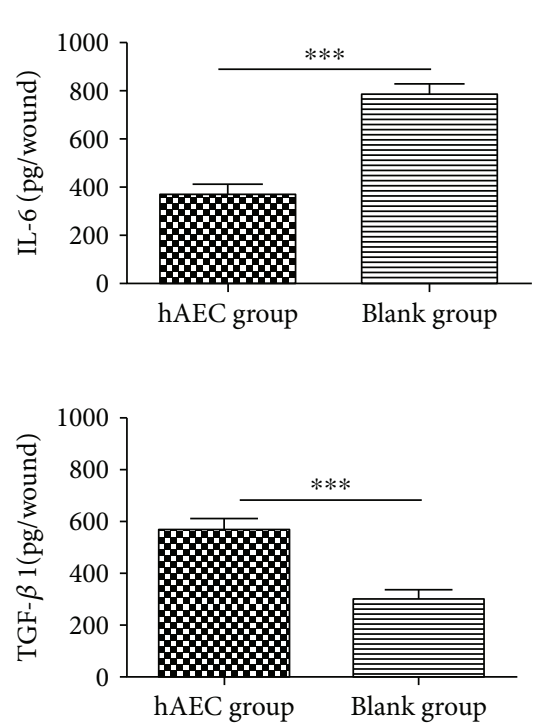
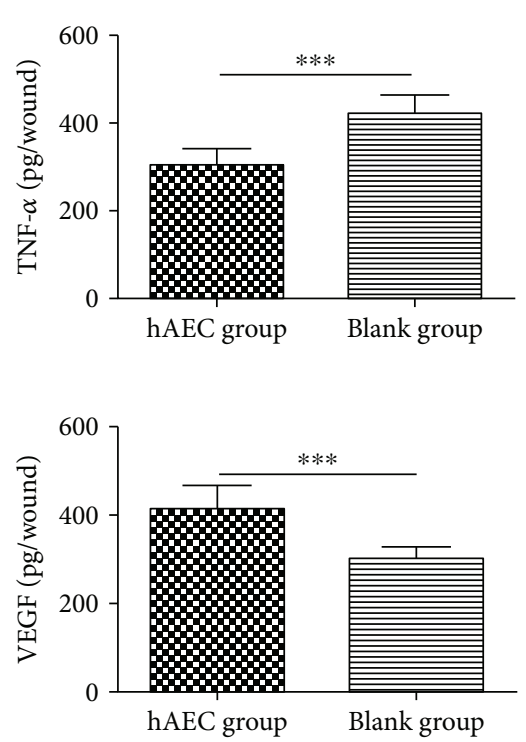

FIGURE 4: hAECs switched proinflammatory status into prohealing status of diabetic wounds. Expression levels of IL-6, IL-1 $\beta$, TNF- $\alpha$, VEGF, TGF- $\beta 1$, and IGF-1 in day 10 diabetic wounds were detected by ELISA, showing reduced levels of proinflammatory cytokines and increased expression levels of prohealing cytokines in the hAEC group. Data was shown as means \pm SD; $n=6 ;{ }^{* * *} P<0.001$. 


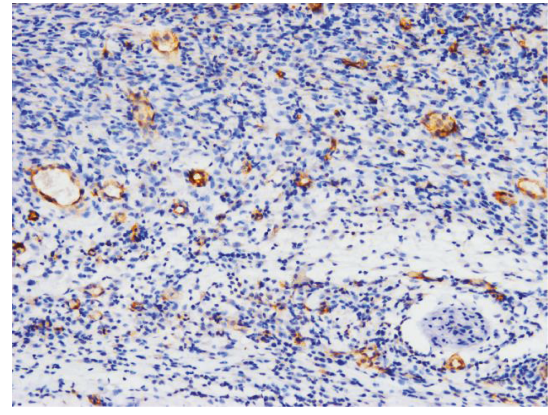

(a)

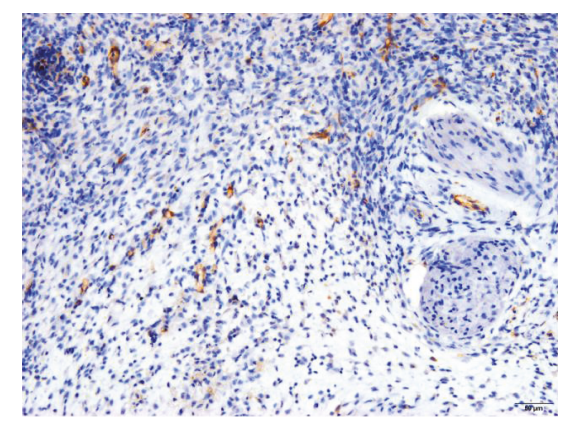

(b)

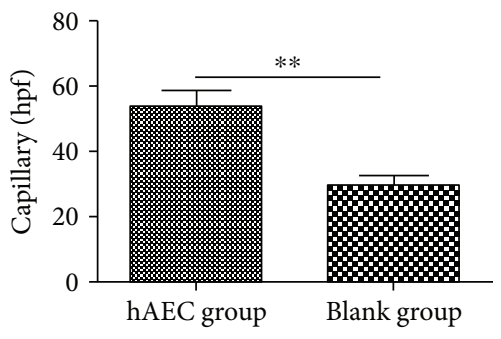

(c)

FIGURE 5: hAECs increased capillary density in diabetic wounds. Representative immunohistochemistry staining of CD31 at day 10 after wounding in the hAEC group (a) and PBS group (b). Statistical analysis of CD $31^{+}$newly formed vessels showed that neovascularization was more obvious in hAEC-treated wounds (c). Data was shown as means \pm SD; $n=6$; ${ }^{* *} P<0.01$; scale bars: $50 \mu \mathrm{m}(\mathrm{a}, \mathrm{b})$.
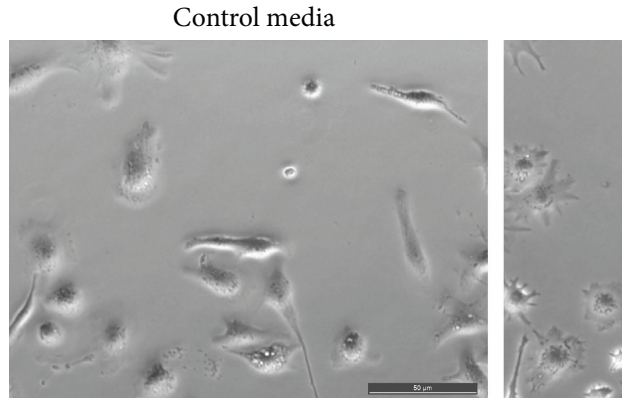

TNF- $\alpha+$ IFN- $\gamma$

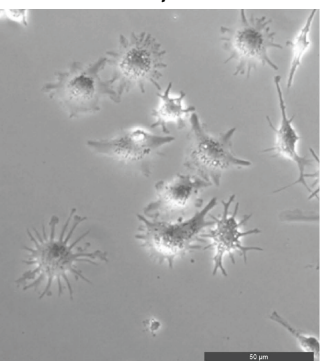

(a)

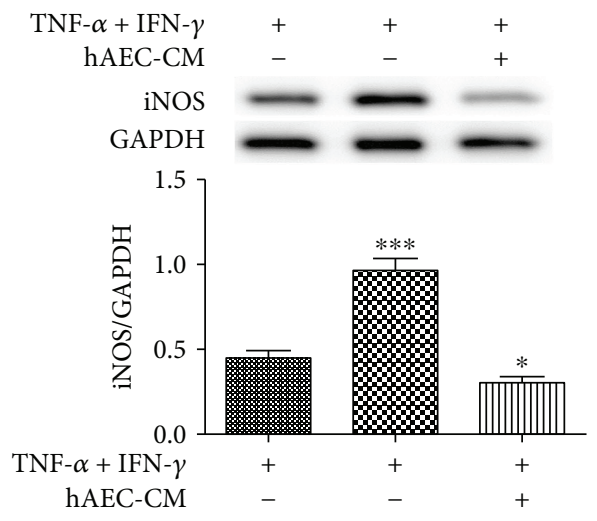

hAEC-CM + TNF- $\alpha+$ IFN- $\gamma$
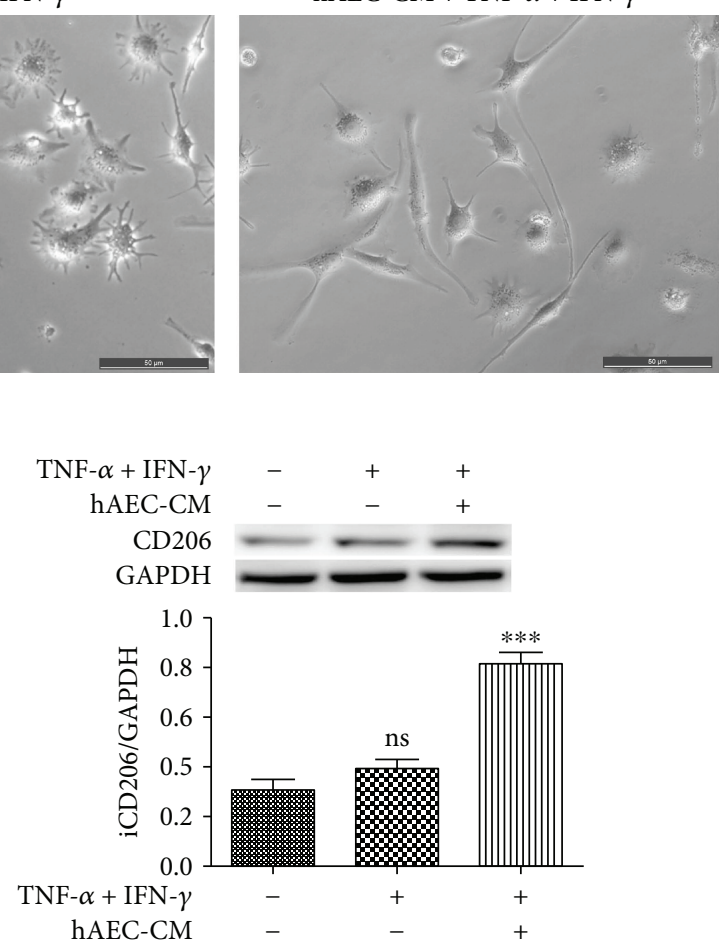

(b)

FIGURE 6: hAEC-CM promoted phenotype switch of macrophages toward M2-like in vitro. (a) Changes of macrophages in morphology when treated with IFN- $\gamma$ and TNF- $\alpha$ with or without hAEC-CM. Complete medium without IFN- $\gamma$ and TNF- $\alpha$ was treated as the control medium. (b) Protein expression levels of iNOS and CD206 when treated with the control medium, IFN- $\gamma+$ TNF- $\alpha$, or IFN- $\gamma+$ TNF- $\alpha+$ hAEC-CM were measured by Western blot. Data was shown as means \pm SD; $n=3 ;{ }^{*} P<0.05 ;{ }^{* * *} P<0.001$; and ns: no significance; scale bars: $50 \mu \mathrm{m}$ (a).

significantly higher in day 10 hAEC-treated wounds than that in PBS-treated wounds (Figure 2(c)).

\section{2. hAECs Promoted Macrophage Phenotype Switch from M1} Macrophage to M2 Macrophage in Diabetic Wounds. The numbers of M1 and M2 macrophages in day 10 wounds were assessed by immunofluorescence (Figure 3(a)). Using iNOS as a marker of M1 macrophage and CD206 as a marker of M2 macrophage, we found that hAEC-treated wounds had fewer M1 macrophages (Figure 3(b)), more M2 macrophages (Figure 3(c)), and a significantly lower M1/M2 ratio (Figure 3(d)). We then measured the expression of inflammatory cytokines and prohealing cytokines in day 10 wounds using ELISA. The expression levels of proinflammatory cytokines IL- $1 \beta$, IL-6, and TNF- $\alpha$ were significantly reduced in the hAEC group, and the levels of prohealing cytokines VEGF, TGF- $\beta 1$, and IGF-1 were significantly increased in the hAEC group when compared with the PBS group 


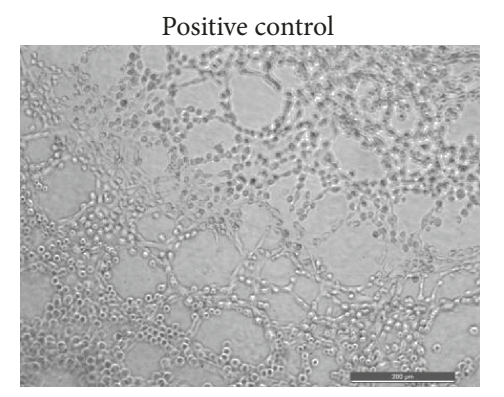

Negative control
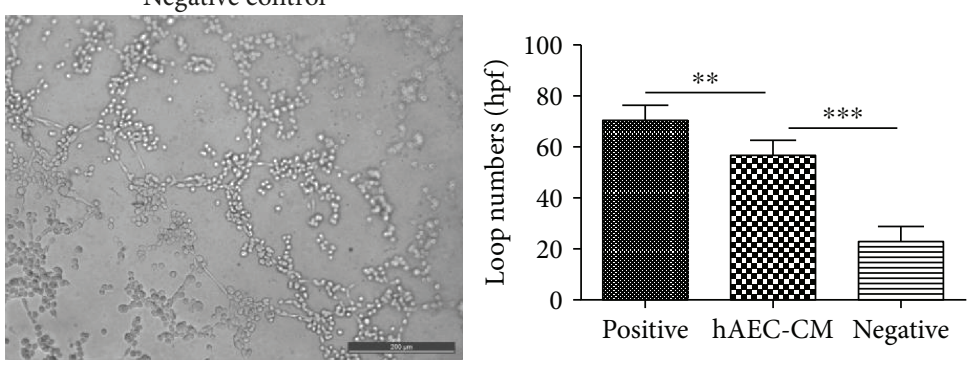

(a)
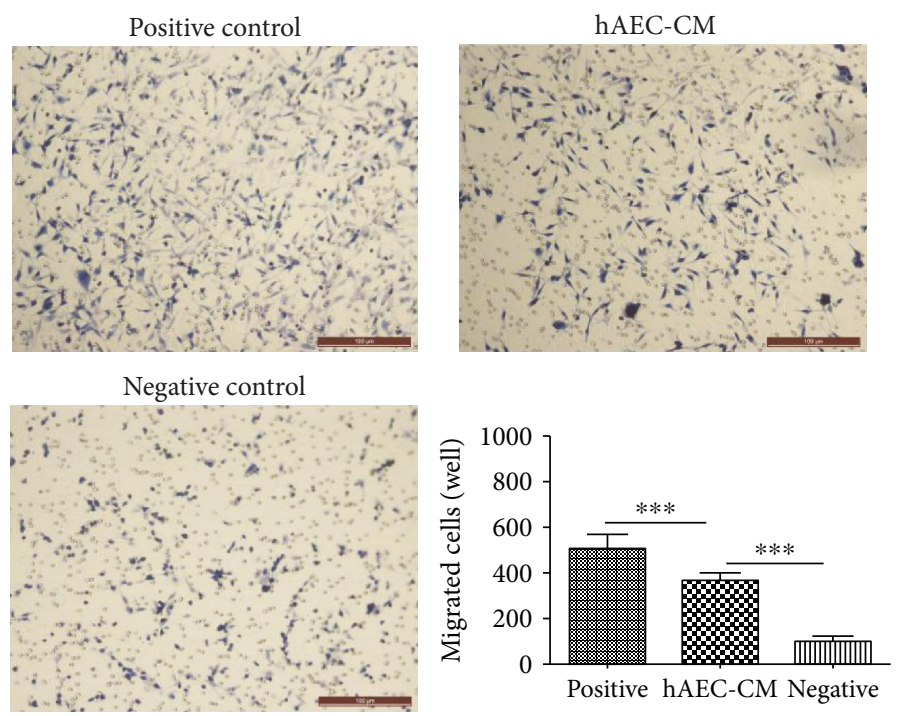

(b)

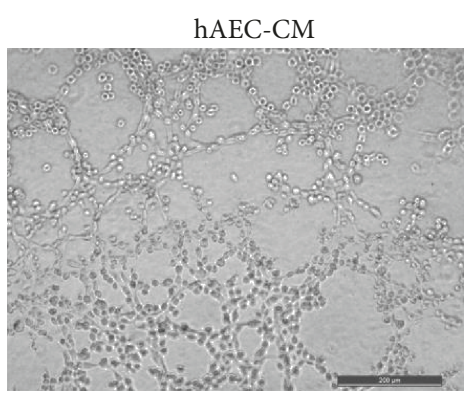

Positive hAEC-CM Negative 


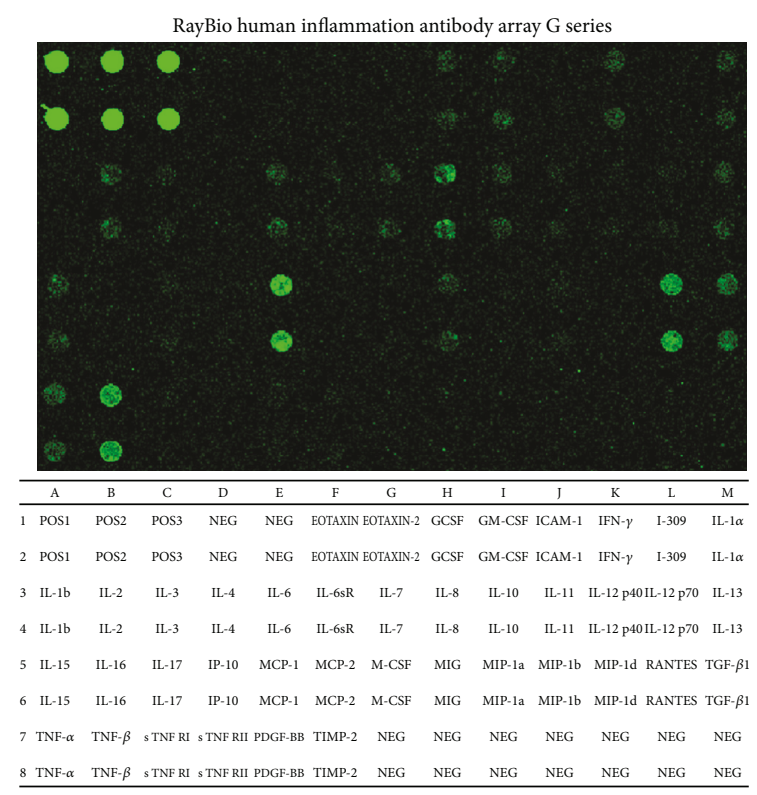

RayBio human inflammation antibody array G series

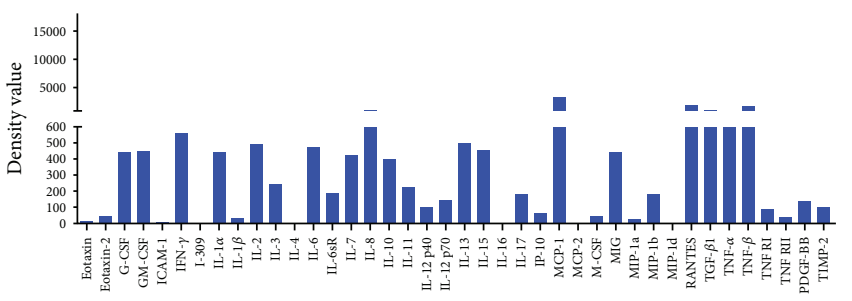

(a)

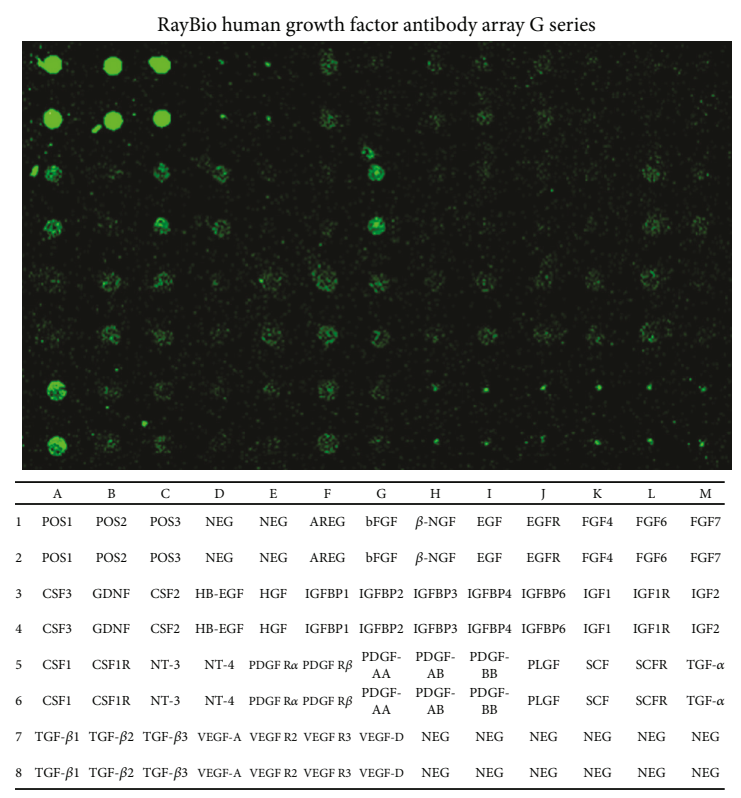

RayBio human growth factor antibody array $\mathrm{G}$ series

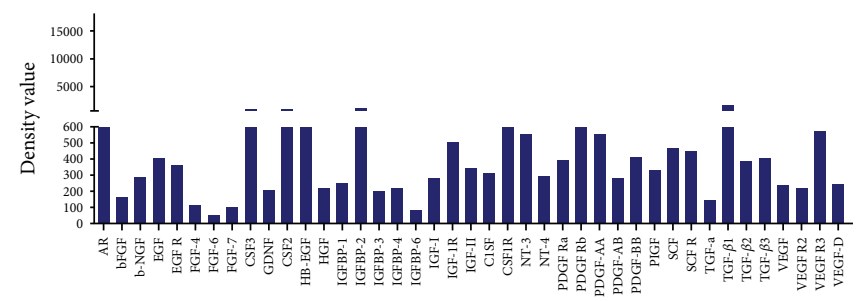

(b)

FIGURE 8: Inflammatory cytokines and growth factors in hAEC-CM. (a) RayBio human inflammatory cytokine antibody array was used to detect a panel of inflammatory cytokines in hAEC-CM. (b) RayBio human growth factor antibody array was used to detect a panel of growth factors in hAEC-CM.

3.5. hAEC-CM Promoted Migration, Proliferation, and Tube Formation of HUVECs In Vitro. The tube formation assay was performed to investigate the effect of hAEC-CM on the angiogenic capacity of HUVECs. We found that more networks formed when treated with hAEC-CM (Figure 7(a)). In addition, hAEC-CM significantly promoted the migration and proliferation of HUVECs performed by transwell assay and CCK-8 assay, respectively (Figures 7(b) and 7(c)).

3.6. Inflammatory Cytokines and Growth Factors in hAEC$C M$. Of the 40 inflammatory cytokines showed in the human inflammation antibody array, IL-8, MCP-1, RANTES, MIP$1 b$, TGF- $\beta 1$, TNF, and IL-13 in hAEC-CM represented the highest expression levels (Figure 8(a)). Of the 41 growth factors represented in the human growth factor antibody array, CSF2, CSF3, HB-EGF, IGFBP-2, CSF1R, PDGF, and TGF- $\beta 1$ in hAEC-CM demonstrated the highest expression levels (Figure 8(b)). The results showed similar kinds of inflammatory cytokines and growth factors representing the higher expression levels with the previous study, which have performed a cytokine array containing 507 human cytokines on the hAEC-derived-conditioned medium [13].

\section{Discussion}

hAECs are nontumorigenic, highly abundant, and low immunogenic and possess multipotent differentiation ability, which make them become ideal alternative stem cell source for regenerative medicine. Previous studies have demonstrated the therapeutic potential of hAECs in various tissue repairs, including lung injury $[10,11,14]$, brain injury [20], myocardial infarction [21], kidney injury [22], and liver fibrosis $[23,24]$. Nonetheless, the therapeutic effect of hAECs on diabetic wound healing is still unknown. In this study, we first reported that hAECs significantly accelerated the diabetic wound healing rate and granulation tissue formation, at least partially, by regulating inflammation and promoting neovascularization.

Macrophage polarization plays an important part in normal wound healing progression. During the inflammatory phase, M1 macrophages initiate an urgent inflammatory response, whereas during the proliferative phase, M2 macrophages accelerate neovascularization and granulation tissue formation [25]. A poorly healing wound such as a diabetic wound is characterized by persevering inflammatory response with protracted aggregation of M1 macrophages 
along with high-level expression of inflammatory factors and low-level expression of prohealing cytokines [26, 27]. Previous studies have shown that promoting macrophage phenotype switch from the M1 macrophage to the M2 macrophage could accelerate diabetic wound healing effectively [28-30]. hAECs could reduce fibrosis and promote repair of damaged tissue by modulating inflammation and promoting macrophage polarization from M1 macrophage to M2 macrophage $[14,31]$, but the effect of hAECs on macrophage phenotype switch in diabetic wounds is at present unknown. In this study, we found that hAECs could promote macrophage phenotype switch from M1 macrophage to M2 macrophage and switch proinflammatory status into prohealing status of diabetic wounds. These results from our in vitro and in vivo experiments are consistent with the previously reported data. Using RayBio human inflammation antibody arrays, we found that hAECs secreted IL-13 and TGF- $\beta 1$, which contributed to the polarization of macrophages [32, 33]. This finding may partly explain the promoting effect of hAECs on macrophage polarization in vitro and in vivo.

Another factor contributing to refractory diabetic wound healing is the inhibited neovascularization [27]. Previous studies have reported that hAECs could promote neovascularization in the damaged tissues, including chemotherapyinduced ovarian damage and hyperoxia-induced lung injury $[13,34]$. However, whether hAECs could promote neovascularization in diabetic wounds is currently unaware. In this study, we found increased capillary density in hAECtreated wounds. Two likely underlying reasons may lead to this result. First, we suspected that the prohealing local environment of the hAEC-treated diabetic wounds associated with increased number of M2 macrophages and elevated levels of various growth factors promoted neovascularization indirectly. Second, our results showed that hAEC-CM could promote proliferation, migration, and tube formation of endothelial cells, suggesting the paracrine effects of hAECs on neovascularization directly. Using RayBio human inflammation antibody arrays and growth factor antibody arrays, we found that a variety of active peptides (PDGF, IL-8, and TGF- $\beta 1$ ) were secreted from hAECs and these peptides are strongly associated with neovascularization [35-37]. This finding may partly explain the direct promoting effect of hAECs on neovascularization.

The present study has its limitation. First, notwithstanding some potential active peptides screened by RayBio antibody arrays may explain the effects of hAECs on the function of macrophages and endothelial cells; further studies are still necessary to find out the key active peptides and illuminate related signaling passway. Second, although the potential mechanisms of hAECs in promoting tissue repairs are not yet well-understood, the main therapeutic mechanisms of stem cells have been explained by paracrine effects rather than transdifferentiation, considering that only a fraction of grafted hAECs could survive in the damaged tissues $[13,20,38]$. Furthermore, impaired diabetic wound healing is characterized by hypoxia, impaired neovascularization, and excessive inflammation, which are extremely detrimental to the survival of hAECs in the wounds. For all the above reasons, it suggests that hAECs are hard to survive in the diabetic wounds for a long period of time and paracrine effects rather than transdifferentiation may play a key role of hAECs in promoting diabetic wound healing. However, further researches are still necessary to provide the direct evidences to determine the fate of hAECs after transplantation onto diabetic wounds.

\section{Conclusions}

Taken together, the results of the present study first showed that hAECs significantly accelerated the diabetic wound healing rate and granulation tissue formation, at least partially, by regulating inflammation and promoting neovascularization. hAECs are nontumorigenic, highly abundant, and low immunogenic, suggesting that it might be a safe and effective therapeutic approach to treat diabetic ulcers and other chronic wounds in clinics.

\section{Data Availability}

The data used to support the findings of this study are available from the corresponding author upon request.

\section{Conflicts of Interest}

The authors declare that there is no conflict of interest regarding the publication of this paper.

\section{Acknowledgments}

This work was funded by the National Natural Science Foundation of China (81701905, 81772076, 81372058, 81571897, and 81501645), the Shanghai Pujiang Program (17PJD043), “Twelfth Five-Year" Scientific Program of China (AWS14C001, 201502028, and AWS11J008), Clinical Key Discipline Project of Shanghai and China, and Shanghai Health System Excellent Talent Training Program (2017BR037).

\section{References}

[1] A. J. M. Boulton, D. G. Armstrong, S. F. Albert et al., "Comprehensive foot examination and risk assessment. A report of the Task Force of the Foot Care Interest Group of the American Diabetes Association, with endorsement by the American Association of Clinical Endocrinologists," Physical Therapy, vol. 88, no. 11, pp. 1436-1443, 2008.

[2] G. Pelizzo, M. A. Avanzini, A. Icaro Cornaglia et al., "Mesenchymal stromal cells for cutaneous wound healing in a rabbit model: pre-clinical study applicable in the pediatric surgical setting," Journal of Translational Medicine, vol. 13, no. 1, p. 219, 2015.

[3] S. E. Hanson, K. R. Kleinbeck, D. Cantu et al., "Local delivery of allogeneic bone marrow and adipose tissue-derived mesenchymal stromal cells for cutaneous wound healing in a porcine model," Journal of Tissue Engineering and Regenerative Medicine, vol. 10, no. 2, pp. E90-E100, 2016.

[4] H. Doi, Y. Kitajima, L. Luo et al., "Potency of umbilical cord blood- and Wharton's jelly-derived mesenchymal stem cells for scarless wound healing," Scientific Reports, vol. 6, no. 1, p. $18844,2016$. 
[5] C. Caliari-Oliveira, J. N. U. Yaochite, L. N. Z. Ramalho et al., "Xenogeneic mesenchymal stromal cells improve wound healing and modulate the immune response in an extensive burn model," Cell Transplantation, vol. 25, no. 2, pp. 201$215,2016$.

[6] S. Wolbank, A. Peterbauer, M. Fahrner et al., "Dose-dependent immunomodulatory effect of human stem cells from amniotic membrane: a comparison with human mesenchymal stem cells from adipose tissue," Tissue Engineering, vol. 13, no. 6, pp. 1173-1183, 2007.

[7] G. Pratama, V. Vaghjiani, J. Y. Tee et al., "Changes in culture expanded human amniotic epithelial cells: implications for potential therapeutic applications," PLoS One, vol. 6, no. 11, article e26136, 2011.

[8] A. Toda, M. Okabe, T. Yoshida, and T. Nikaido, "The potential of amniotic membrane/amnion-derived cells for regeneration of various tissues," Journal of Pharmacological Sciences, vol. 105, no. 3, pp. 215-228, 2007.

[9] S. Murphy, S. Rosli, R. Acharya et al., "Amnion epithelial cell isolation and characterization for clinical use," Current Protocols in Stem Cell Biology, vol. 13, no. 1, pp. 1E.6.1-1E.6.25, 2010.

[10] J. L. Tan, Y. Z. Tan, R. Muljadi et al., "Amnion epithelial cells promote lung repair via lipoxin A4," Stem Cells Translational Medicine, vol. 6, no. 4, pp. 1085-1095, 2017.

[11] S. Murphy, R. Lim, H. Dickinson et al., "Human amnion epithelial cells prevent bleomycin-induced lung injury and preserve lung function," Cell Transplantation, vol. 20, no. 6, pp. 909-924, 2011.

[12] Q. Yang, M. Luo, P. Li, and H. Jin, "Transplantation of human amniotic epithelial cells repairs brachial plexus injury: pathological and biomechanical analyses," Neural Regeneration Research, vol. 9, no. 24, pp. 2159-2163, 2014.

[13] Q. Zhang, S. Bu, J. Sun et al., "Paracrine effects of human amniotic epithelial cells protect against chemotherapyinduced ovarian damage," Stem Cell Research \& Therapy, vol. 8, no. 1, p. 270, 2017.

[14] J. L. Tan, S. T. Chan, E. M. Wallace, and R. Lim, "Human amnion epithelial cells mediate lung repair by directly modulating macrophage recruitment and polarization," Cell Transplantation, vol. 23, no. 3, pp. 319-328, 2014.

[15] D. Baltzis, I. Eleftheriadou, and A. Veves, "Pathogenesis and treatment of impaired wound healing in diabetes mellitus: new insights," Advances in Therapy, vol. 31, no. 8, pp. 817836, 2014.

[16] S. C. Bryer, G. Fantuzzi, N. Van Rooijen, and T. J. Koh, "Urokinase-type plasminogen activator plays essential roles in macrophage chemotaxis and skeletal muscle regeneration," Journal of Immunology, vol. 180, no. 2, pp. 1179-1188, 2008.

[17] Y. Zhang, X. Liang, S. Liao et al., "Potent paracrine effects of human induced pluripotent stem cell-derived mesenchymal stem cells attenuate doxorubicin-induced cardiomyopathy," Scientific Reports, vol. 5, no. 1, article 11235, 2015.

[18] N. S. Tan and W. Wahli, "Studying wound repair in the mouse," Current Protocols in Mouse Biology, vol. 3, no. 3, pp. 171-185, 2013.

[19] Y. Zheng, X. Wang, S. Ji et al., "Mepenzolate bromide promotes diabetic wound healing by modulating inflammation and oxidative stress," American Journal of Translational Research, vol. 8, no. 6, pp. 2738-2747, 2016.
[20] B. Leaw, D. Zhu, J. Tan et al., "Human amnion epithelial cells rescue cell death via immunomodulation of microglia in a mouse model of perinatal brain injury," Stem Cell Research \& Therapy, vol. 8, no. 1, p. 46, 2017.

[21] Y. S. Song, H. W. Joo, I. H. Park et al., "Transplanted human amniotic epithelial cells secrete paracrine proangiogenic cytokines in rat model of myocardial infarction," Cell Transplantation, vol. 24, no. 10, pp. 2055-2064, 2015.

[22] J. Liu, R. Hua, Z. Gong et al., "Human amniotic epithelial cells inhibit CD4+ T cell activation in acute kidney injury patients by influencing the miR-101-c-Rel-IL-2 pathway," Molecular Immunology, vol. 81, pp. 76-84, 2017.

[23] A. Hodge, D. Lourensz, V. Vaghjiani et al., "Soluble factors derived from human amniotic epithelial cells suppress collagen production in human hepatic stellate cells," Cytotherapy, vol. 16, no. 8, pp. 1132-1144, 2014.

[24] R. Lim, A. Hodge, G. Moore, E. M. Wallace, and W. Sievert, "A pilot study evaluating the safety of intravenously administered human amnion epithelial cells for the treatment of hepatic fibrosis," Frontiers in Pharmacology, vol. 8, p. 549, 2017.

[25] S. Gordon and F. O. Martinez, "Alternative activation of macrophages: mechanism and functions," Immunity, vol. 32, no. 5, pp. 593-604, 2010.

[26] R. Blakytny and E. Jude, "The molecular biology of chronic wounds and delayed healing in diabetes," Diabetic Medicine, vol. 23, no. 6, pp. 594-608, 2006.

[27] V. Falanga, "Wound healing and its impairment in the diabetic foot," The Lancet, vol. 366, no. 9498, pp. 1736-1743, 2005.

[28] R. E. Mirza, M. M. Fang, W. J. Ennis, and T. J. Koh, "Blocking interleukin- $\beta$ induces a healing-associated wound macrophage phenotype and improves healing in type 2 diabetes," Diabetes, vol. 62, no. 7, pp. 2579-2587, 2013.

[29] E. C. Leal, E. Carvalho, A. Tellechea et al., "Substance P promotes wound healing in diabetes by modulating inflammation and macrophage phenotype," The American Journal of Pathology, vol. 185, no. 6, pp. 1638-1648, 2015.

[30] A. Tellechea, E. C. Leal, A. Kafanas et al., "Mast cells regulate wound healing in diabetes," Diabetes, vol. 65, no. 7, pp. 2006-2019, 2016.

[31] U. Manuelpillai, D. Lourensz, V. Vaghjiani et al., "Human amniotic epithelial cell transplantation induces markers of alternative macrophage activation and reduces established hepatic fibrosis," PLoS One, vol. 7, no. 6, article e38631, 2012.

[32] J. R. Grunwell, S. M. Yeligar, S. Stephenson et al., “TGF- $\beta 1$ suppresses the type I IFN response and induces mitochondrial dysfunction in alveolar macrophages," The Journal of Immunology, vol. 200, no. 6, pp. 2115-2128, 2018.

[33] S. Gordon, "Alternative activation of macrophages," Nature Reviews Immunology, vol. 3, no. 1, pp. 23-35, 2003.

[34] D. Zhu, R. Muljadi, S. T. Chan et al., "Evaluating the impact of human amnion epithelial cells on angiogenesis," Stem Cells International, vol. 2016, Article ID 4565612, 13 pages, 2016.

[35] K. T. Bauerle, R. E. Schweppe, G. Lund et al., "Nuclear factor $\kappa \mathrm{B}$-dependent regulation of angiogenesis, and metastasis in an in vivo model of thyroid cancer is associated with secreted interleukin-8," The Journal of Clinical Endocrinology \& Metabolism, vol. 99, no. 8, pp. E1436-E1444, 2014.

[36] A. Dong, C. Seidel, D. Snell et al., "Antagonism of PDGF-BB suppresses subretinal neovascularization and enhances the 
effects of blocking VEGF-A," Angiogenesis, vol. 17, no. 3, pp. 553-562, 2014.

[37] A. K. Wara, S. Foo, K. Croce et al., “TGF- $\beta 1$ signaling and Krüppel-like factor 10 regulate bone marrow-derived proangiogenic cell differentiation, function, and neovascularization," Blood, vol. 118, no. 24, pp. 6450-6460, 2011.

[38] P. Kuchroo, V. Dave, A. Vijayan, C. Viswanathan, and D. Ghosh, "Paracrine factors secreted by umbilical cordderived mesenchymal stem cells induce angiogenesis in vitro by a VEGF-independent pathway," Stem Cells and Development, vol. 24, no. 4, pp. 437-450, 2015. 


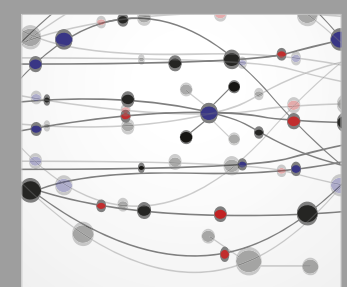

The Scientific World Journal
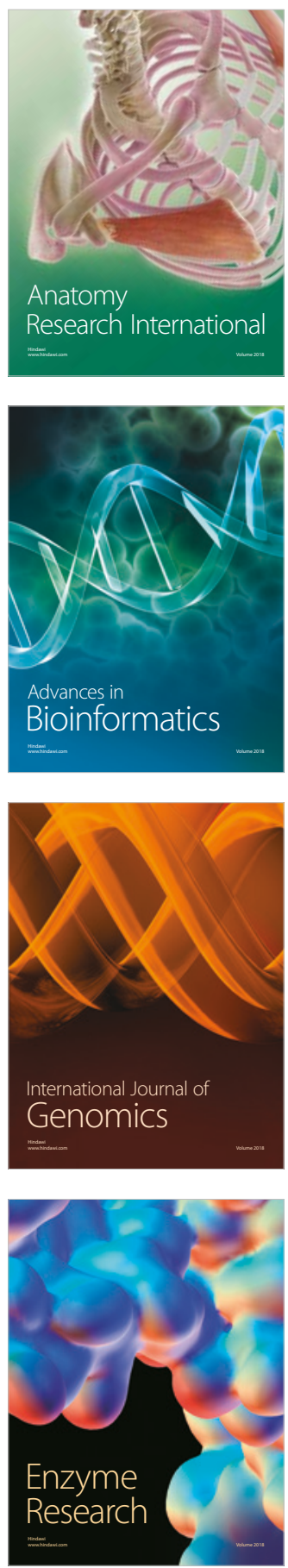
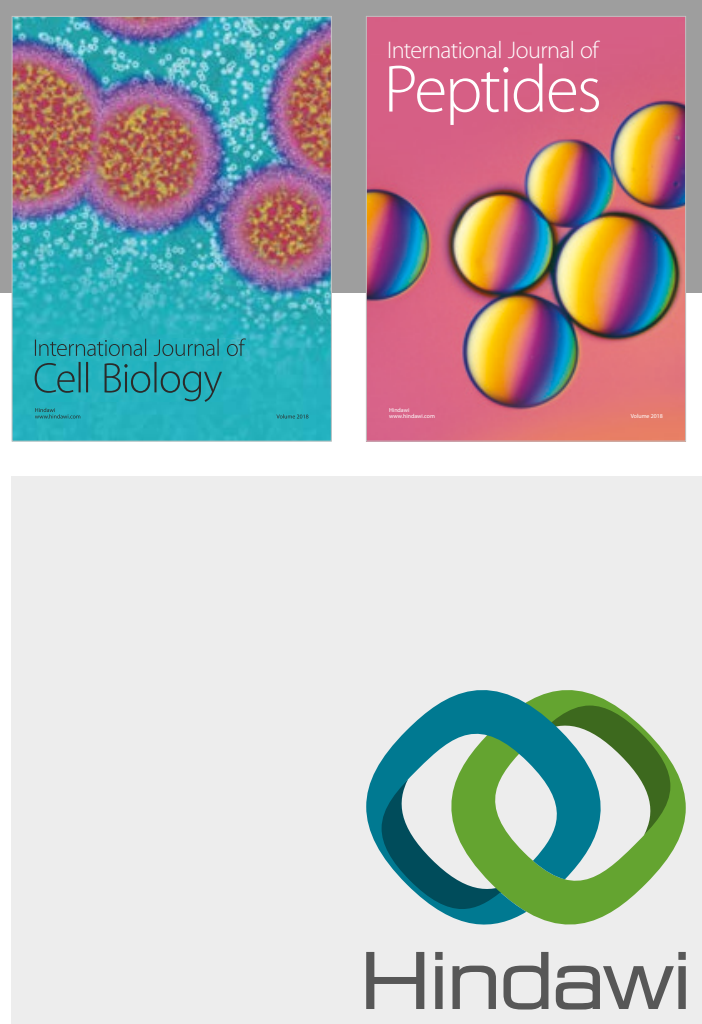

Submit your manuscripts at

www.hindawi.com
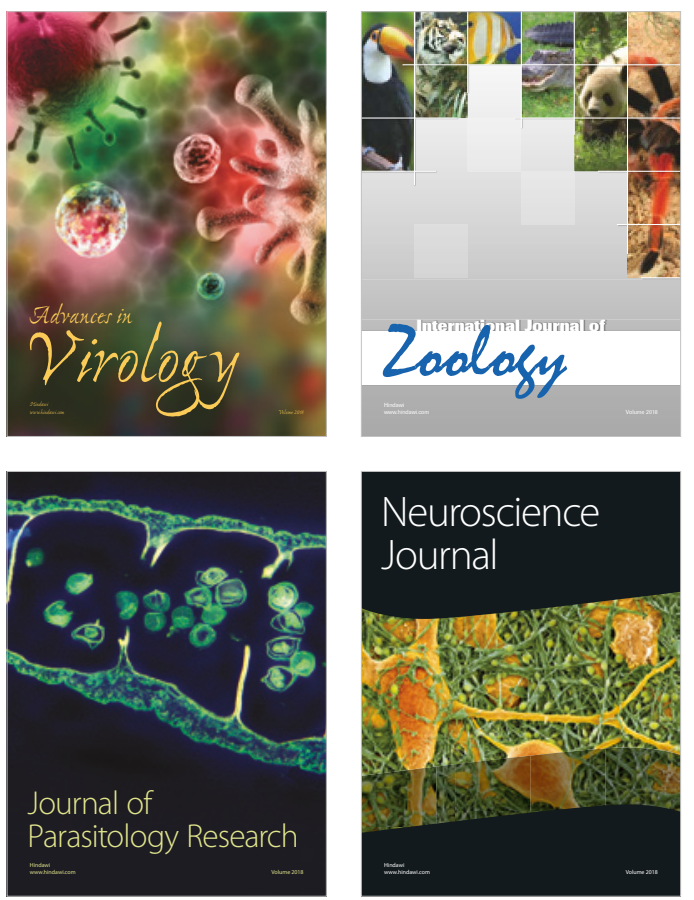
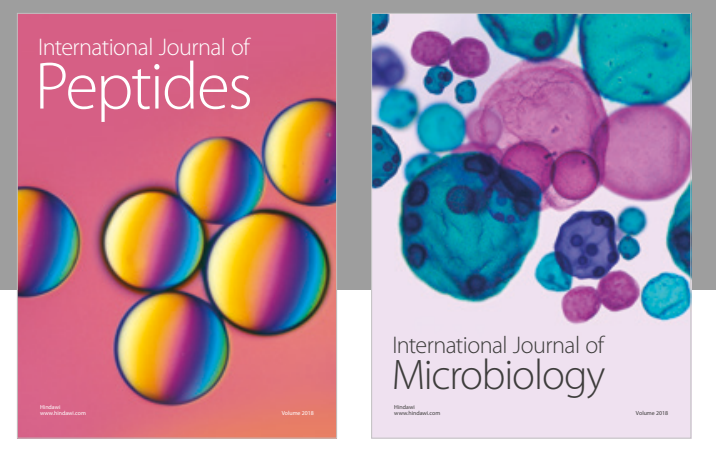

nternational Journal of Microbiology
Journal of
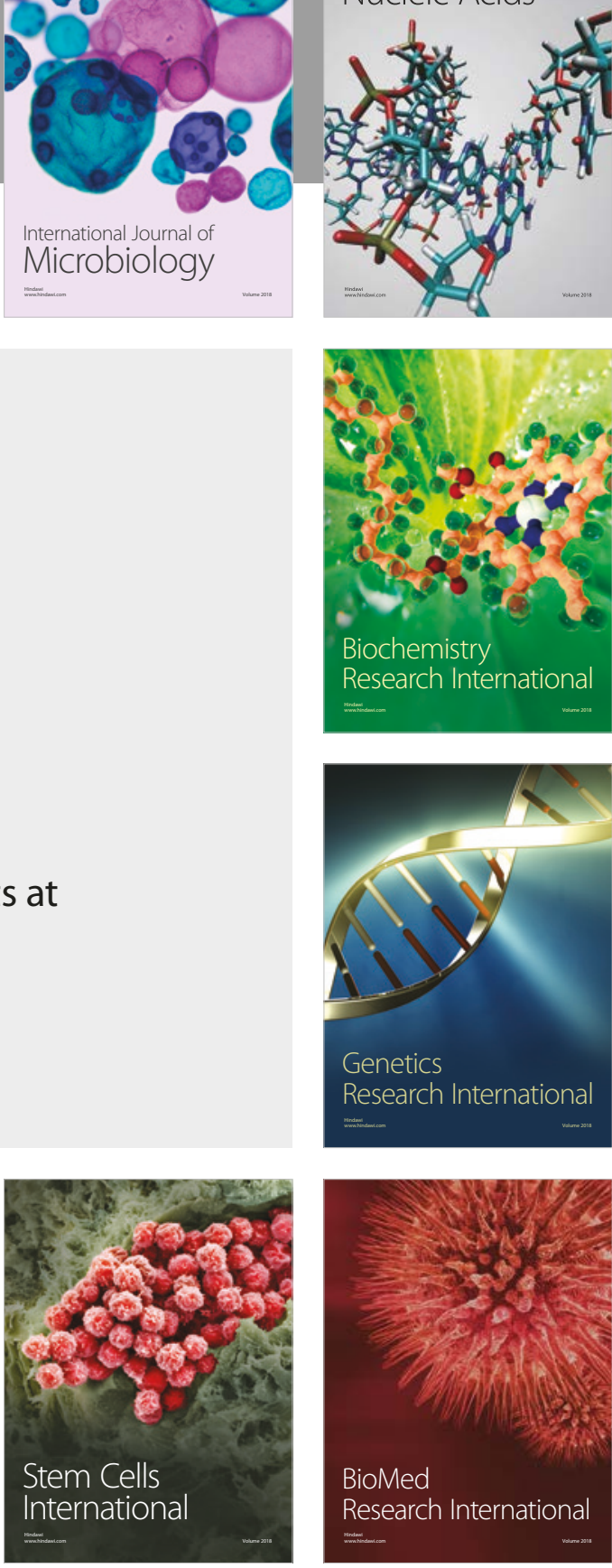
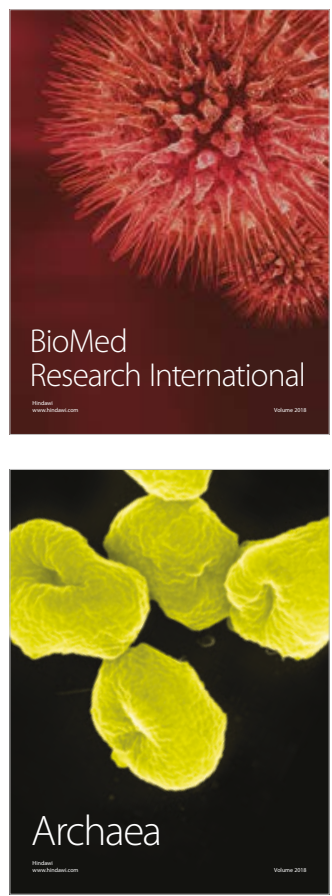